\title{
Application of hydrogels on highly polluted affluents: the leachate
}

\author{
R. Cioffi, C. Ferone, G. Perillo \& F. Sorrentino \\ Department of Technology, University of Naples Parthenope, Italy
}

\begin{abstract}
Hydrogels are a class of compounds formed of colloidal polymer chains of molecules dispersed in water, which have a high absorbent capacity and can be classified as biodegradable and biocompatible. The absorbent capacity of hydrogels is such that they can engage a quantity of liquid equal to approximately one thousand times their own weight. It is estimated, therefore, that one kilogram of this material can absorb up to one ton of water. In addition, hydrogels are able to change the physical appearance of the absorbed liquid by including its volatile components in their starting matrix so that the latter are immobilized and not dispersed into the atmosphere, which offers considerable advantages from the environmental point of view.

In a previous paper, applicative potentialities have been evaluated. In the present paper, not only molecular structure of the polymer and its reactivity with water have been investigated, but also its use in the environmental field. In fact, thanks to available data carried out during the previous study, laboratory tests have been developed in order to define the specific retention capacity exert against aqueous solutions containing contaminating substances, as leachate. Keywords: hydrogels, liquid contaminants, leachate.
\end{abstract}

\section{Introduction}

The characteristics of hydrogels make them suitable for application in a number of different fields, such as waste treatment, the remediation of contaminated sites in order to minimise the flow and the underground migration of liquid contaminants, and interventions for the purification of contaminated waters [1].

The objective of this paper is to evaluate the potential applications of some polymer blends, known as hydrogels, which are capable of absorbing large 
quantities of liquids within their matrix, changing their physical appearance and transforming them into gel. They also absorb highly volatile substances, immobilizing them and preventing their release into the atmosphere.

The field of application considered is waste treatment, starting from the remediation of contaminated sites and including the requalification of landfills in order to stem the flow and underground migration of liquid contaminants, such as leachate, the management of refuse-derived fuel bales, and ensuring the safety of leachate collection sites, waste collection vehicles and public dumpsters. An important feature of these polymers lies in their ability to absorb liquids within a saturated porous medium, which makes them suitable for use underground [2].

During the active life of a landfill, and to a lesser degree after its closure, water falling on the site passes through the waste giving rise to leachate. The quantity of leachate formed is dependent on the landfill's location while its quality is determined by the type of waste. In order to prevent the accumulation of leachate in the bottom of the landfill, it is channelled, collected and removed for subsequent purification treatments. A leachate collection and drainage system in a properly managed landfill can be made from natural materials (sand and gravel) or synthetic materials, but such systems are often subject to leaks caused by unnoticed breaks in the impermeable lining or defective welds in the geomembrane, making them inefficient.

Finally, super-absorbent polymers have been considered to realise "pad", containing within the superabsorbent polymer mixtures, in order to reset the percolation coming from the MSW masses.

\section{Materials and methods}

\subsection{Materials}

The gels considered are almost always obtained from suitably reticulated mixtures of sodium carboxylmethyl cellulose $(\mathrm{CMCNa})$ and hydroxyethyl cellulose (HEC) [3]. The structure of the repetitive units of CMCNa and HEC is reported in Figure 1.

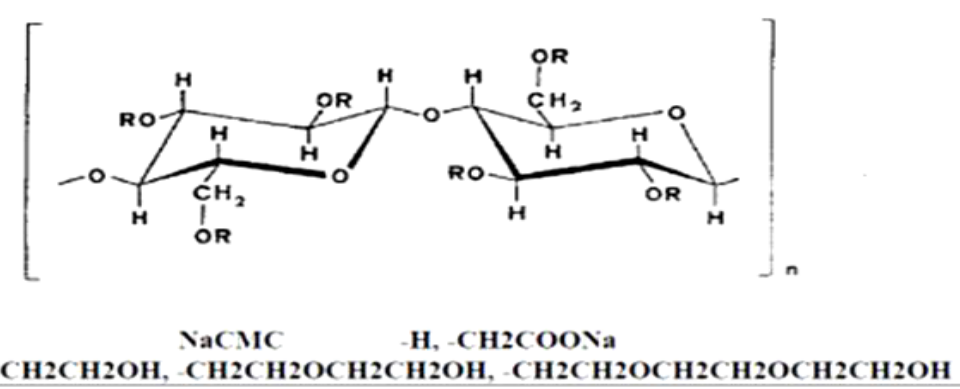

Figure 1: Chemical structure of the cellulose-based macromolecule. 
Our sample, composed of CMCNa and HEC, was checked using FT-IR analysis (Figure 2).

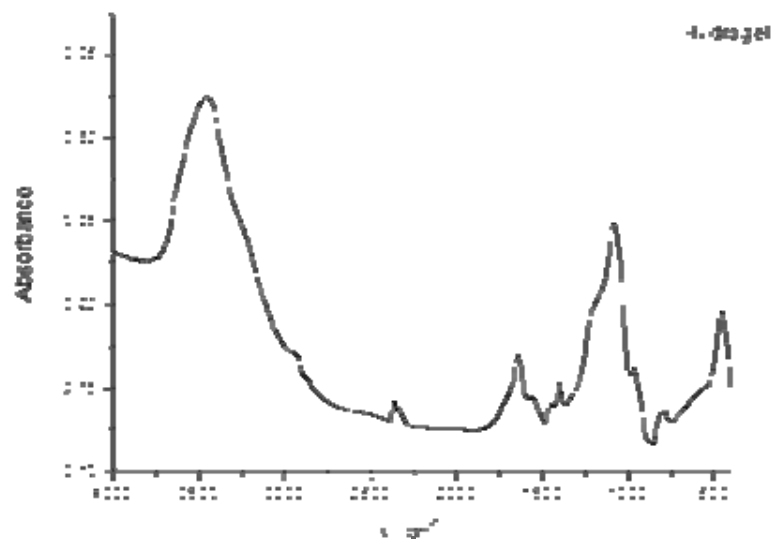

Figure 2: IR graph of the sample.

The fundamental characteristics that a super-absorbent polymer must possess are a high affinity for water, an ionic polymer macromolecule and biodegradability. The latter characteristic is an indispensable requirement for some applications.

Once reticulated, the carboxylmethyl cellulose polymer possesses all the characteristics needed for use as a super-absorbent material. It is derived from cellulose, it is biodegradable and, thanks to the presence of the ionic carboxylmethyic group through an ether bonding with the cellulose structure, it possesses the polyelectrolytic characteristics needed to develop a Donnan equilibrium with the external solution with which it comes into contact. Furthermore, CMC is soluble in water and therefore has a high affinity for it [4, $5]$.

Hydroxyethyl cellulose is also derived from cellulose and has a high affinity for water but, unlike $\mathrm{CMC}$, it does not possess charge groups anchored to the polymer network and, therefore, does not make any contribution to the Donnan effect which, as mentioned above, favours absorption.

\subsection{Synthesis procedures}

The hydrogel is prepared in aqueous solution as it is the contact between the pure solid substance and distilled water that transforms it into a gel and this is the environment in which gelification takes place. In this phase, therefore, the gel is in a swollen state, even though it has not yet reached a swelling value that represents a state of equilibrium. The gel thus obtained is then purified to eliminate any residual impurities [6].

Purification is achieved by placing the gel in distilled water, which is frequently changed until the gel reaches its maximum degree of swelling (the 
material hardens and assumes the shape of the container is which it was placed). The volume of water required for the washes is not less than ten-fifteen times the volume of the gel to be purified after synthesis [7].

In order to assess the material's efficacy in terms of absorbency, it is then necessary to dry the completed and purified gel. Drying can be carried out in three ways:

a) at atmospheric pressure: this simply entails leaving the sample in the air at a temperature of approximately $20^{\circ} \mathrm{C}$ until it dries completely. The time required for this depends on the sample size; this can reasonably be estimated at not less than five days. The sample has a rubbery consistency when swollen, while in the dry state it has a glassy or crystalline consistency. After 5 days the samples show no variation in weight but a nonsignificant variation in volume.

b) in a vacuum: the sample is placed in a vacuum until it has dried and assumes a structure similar to cellulose fluff. The time needed for drying is approximately 5 days, after which time the sample displays a significant reduction in volume (approximately 30\%) and in weight (approximately $5 \%$ ).

c) by extraction with acetone: the sample is placed in a beaker with a $20 \%$ addition of acetone and is subjected to magnetic stirring; the sample is seen to gradually decrease in volume until it reaches an acetone-water concentration achieved following the release of water by the contracting sample.

The gel and acetone-water mixture is then filtered using medium porosity filter paper and the filtered sample is then placed back in the beaker with new pure acetone and is subjected again to magnetic stirring. The procedure is repeated until the sample has eliminated most of the water it contains, significantly and visibly reducing in volume, after which the gel is placed in a $50^{\circ} \mathrm{C}$ oven to eliminate the residual water still trapped in the gel.

As will be seen below, the drying procedure exerts a considerable influence on the material's structure and absorbency.

\subsection{Absorption properties}

The study of the absorption capacity of hydrogels synthesised from polysaccharide polymers focuses on the effect of variations both in the characteristics of the solution with which the gel is brought into contact and in the network's structural parameters [8].

Particular attention is dedicated to the influence of external mechanical stresses on the material's ability to absorb and retain water and aqueous solutions.

The absorption analyses were set up in order to assess the influence of the external solution in contact with the gel on its swelling capacity. This analysis was performed while also varying the material's physico-chemical parameters and comparing the results obtained for the various types of gel. 
In order to evaluate the absorption capacity of the various samples, the swelling ratio parameter $(S . R .=$ swollen sample weight/dry sample weight $)$ was used.

\subsubsection{Absorption properties with water}

Analysis of the absorption capacity in distilled water was repeated and the results were studied for reference purposes. This analysis was conducted in different water vapour activities and for different types of material, making it possible to determine the gel's Flory-Huggins interaction parameter $(\chi)$.

The absorption tests in distilled water and aqueous solutions were carried out in accordance with the following procedure: the gel sample, previously weighed and dried in acetone, is immersed in the solution in which the absorption capacity is to be analysed. The gel is then left to swell until it reaches equilibrium $(\approx 24$ hours $)$.

Once swollen, the gel is removed from the solution and left to drain so as to eliminate excess, unabsorbed water on its surface. The sample is then reweighed.

The differential water absorption experiments in the activity range 0-0.95 for the unreticulated polymer dried at atmospheric pressure and for the reticulated polymer dried in acetone were carried out with the aim of characterising the absorption kinetics as a function of external activity [9].

The absorption tests enable an experimental determination of the absorption isotherm, which in turn makes it possible to obtain information on the interactions between the water and the polymer's hydrophilous groups and, in particular, to determine the Flory-Huggins interaction parameter.

The drying procedure has a major influence on the material's absorption capacity: the highest swelling ratio was observed for gels dried in acetone and the lowest for those dried in the atmosphere.

Differences in morphology can be clearly seen: the sample dried in the atmosphere is dense and compact, the one dried in acetone appears characterised by a more compacted or microporous structure, while the gel dried in vacuum conditions displays an intermediate structure.

The differences in absorption capacity can therefore be attributed to the different morphology of the material: the structures characterised by larger micropores show a higher absorption capacity due to the fact that some of the water condenses in the micropores, which further increase in size as the gel swells.

The drying procedure affects the gel's physical structure and the samples dried with acetone show a higher porosity than those dried in vacuum or atmospheric conditions. Consequently, the effects of capillarity in the swelling mechanism make an essential contribution to absorption and the greater the sample's porosity, the higher its swelling capacity.

\subsubsection{Absorption properties with leachate}

In order to assess the polymers' applicability in environmental remediation, absorption tests were carried out with landfill leachate - a liquid that is created 
primarily by the infiltration of water into masses of municipal solid waste and by the latter's decomposition processes. The tests were conducted on leachate from the municipal solid waste landfill in Terzigno (Naples), a site which is no longer in use.

Analysis of the leachate shows it to be a brown liquid with a typically unpleasant smell that may even be nauseating and extremely difficult to bear, and with a $\mathrm{pH}$ of 9 ; this indicates that the leachate is in an ageing phase and therefore with a low concentration of metals, as high $\mathrm{pH}$ values do not favour their solubilisation. As already mentioned, these tests were conducted in order to determine the polymers' specific absorption capacity for landfill leachate.

The tests aimed only to determine the polymers' nominal absorption capacity in a free state, i.e. left free to swell and expand without any limitation.

The equipment used in the tests consists of a graduated $100 \mathrm{ml}$ beaker and two-significant-figures precision scales. The tests entailed placing a given quantity of leachate in the beaker, starting from $10 \mathrm{ml}$, and adding $1 \mathrm{~g}$ of hydrogel until the absorbent material reaches its maximum retention capacity. The subsequent tests then aimed to determine the maximum quantity of leachate that can be absorbed in free-state conditions by using $1 \mathrm{~g}$ of polymers with the gradual addition of leachate until it reaches full saturation.

The experimental tests showed that the polymers ensure a more than satisfactory absorption capacity and also produce a significant reduction of the unpleasant and malodorous smell released by the leachate [7-9].

The acetone drying test was then performed to determine product reversibility and the possibility for its subsequent reuse as a superabsorbent material. This test was carried out on three samples (1:10,1:20 and 1:30) which, after developing the entire equilibrium reaction, were inserted in a beaker with a quantity of acetone and subjected to magnetic stirring. Finally, the acetone-leachate solution was filtered and the sample placed in a $50^{\circ} \mathrm{C}$ oven for approximately 12 hours. The samples were then seen to return to a semi-pure or glassy state and a second absorption test was carried out with new quantities of leachate.

Moreover, the hydrogel can be used numerous times as a superabsorbent material since its absorption capacity is not extinguished; this constitutes an important solution to the problem of its disposal [10].

The results of the absorption tests indicate that, up to a ratio of $1: 30$, the polymers are able to fully absorb the leachate without leaving any liquid residue. Whereas, with a ratio of 1:40 part of the leachate remains in a liquid state even after long periods of contact with the hydrogel [11].

The absorption capacity of superabsorbent polymers can be expressed as a function of the degree of swelling and summarised in the following graphs (the results are reported in Figure 3 and Figure 4 below).

Overall, the polymers' specific absorption capacity for leachate (approximately $1: 30$ ) is significantly lower than that recorded using demineralised water (approximately 1:100).

This reduction in absorption capacity is most probably determined by chemical interactions between the polymers and the numerous substances present in the leachate. 

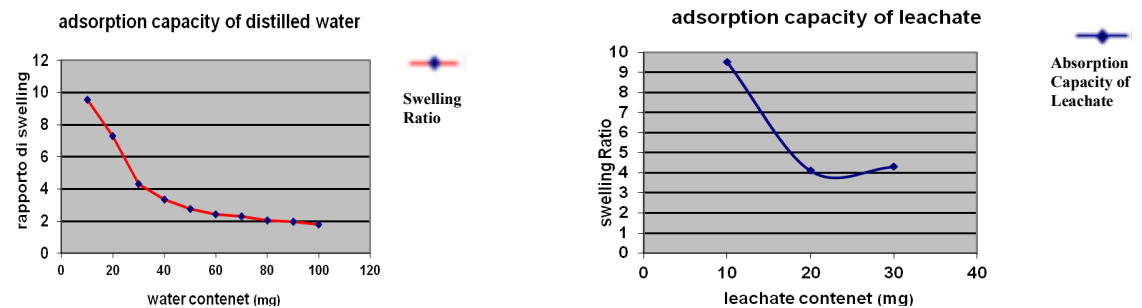

Figure 3: Absorption capacity as a function of degree of swelling.

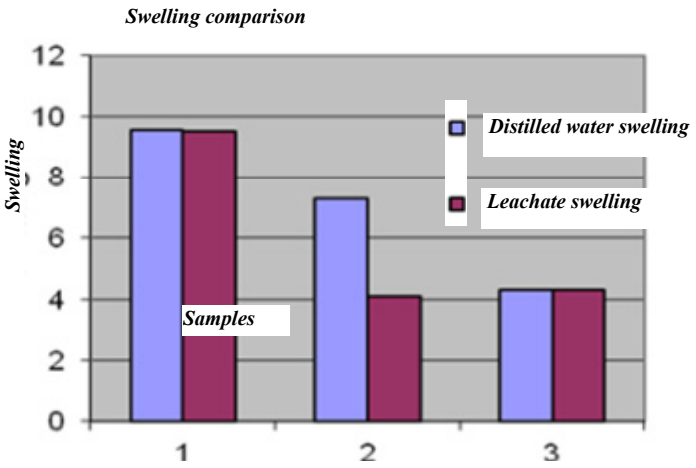

Figure 4: Comparison of the absorption capacity in terms of swelling between leachate and distilled water.

On the other hand, the isotropic compression tests made it possible to determine the quantity of liquid released by this type of polymers when under stress. The results are reported in Figure 5 and Figure 6 below.

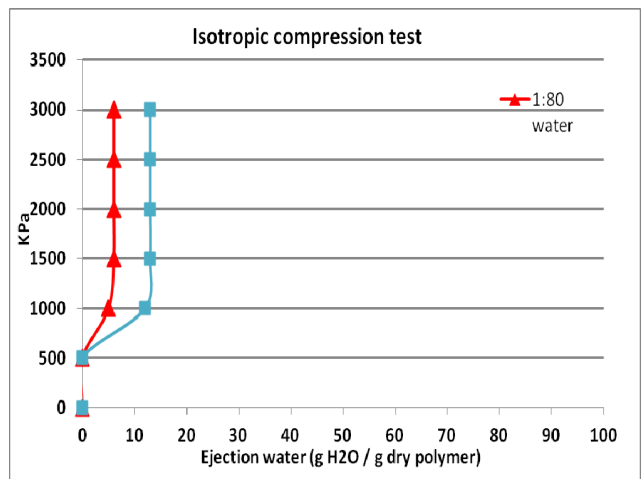

Figure 5: Result of the isotropic compression test, expressed as a function of water released, for the samples 1:50, 1:80 and 1:100 of hydrogel and distilled water. 


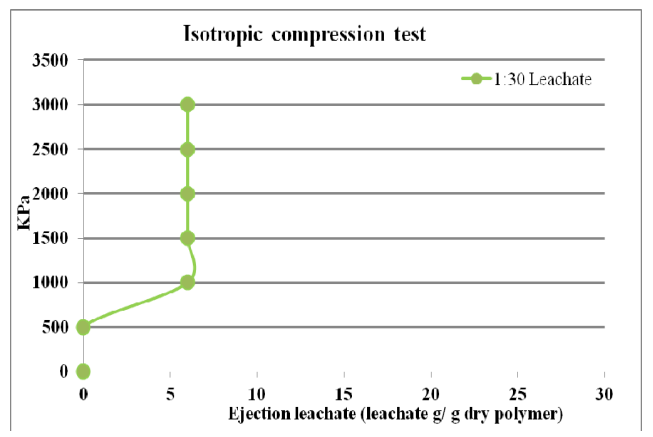

Figure 6: Result of the isotropic compression test, expressed as a function of leachate released, for the samples 1:20 and 1:30 of hydrogel and leachate.

These graphs show that, as the pressure of isotropic compression increases, the sample is no longer able to release liquid because the water or leachate remain trapped in the reticular structure of the superabsorbent polymer.

The isotropic compression test on the sample 1:20 (hydrogel and leachate) points out that it releases no leachate under pressure; and the isotropic compression test on the sample 1:50 (hydrogel and distilled water) points out that it releases no liquid under pressure.

In conclusion, it is possible to estimate that the best concentration ratio between hydrogel and contaminated effluent is 1:20.

\section{Conclusions}

Objective of this research was the creation of a "pad" containing within the superabsorbent polymer blends, with a core made from a double layer of fluff pulp of cellulose, in order to increase the absorbency (Figure 7).
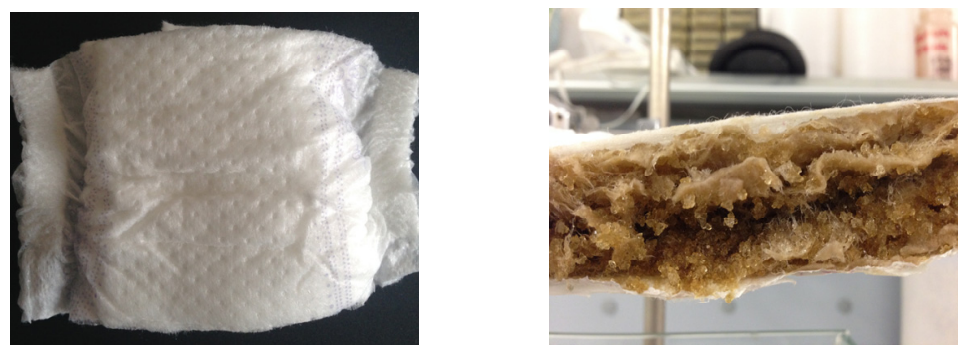

Figure 7: Superabsorbent "pad" model.

The cotton layers, in fact, make the swelling pad be contained in certain limits, in order to avoid problems of leakage of liquid absorbed by the hydrogels. The fluff, made from wood and generated by cellulose fibers, is treated with optical brighteners in order to obtain the maximum degree of absorbency. 
Indeed, the cotton and/or cellulose can absorb water up to twenty-seven times Hydrogels (white microgranules with polymer base) are positioned in the basal zone of the cellulose fluff, where maximum amount of liquid is collected.

The ratio of the above mentioned materials is 1 gram of hydrogel every 6 grams of cellulose fluff.

A non-woven tissue (NWT), Spunbond type, was used as the upper diffusion layer. The Spunbond is made from $100 \%$ polypropylene filaments, randomly arranged and thermally welded. Finally, the external coating was made from a polyethylene film, which acts as a containment barrier for the fluid, combined with a layer of NWT.

Then, a test of adsorption has been made, by means of spilling a known quantity of leachate over our pad.

From the above mentioned test, the maximum absorption value has been determined for a pad of size 30x11, containing 3 grams of hydrogel and 18 of cellulose fluff (respecting the ratio 1:6): $400 \mathrm{ml}$ of leachate.

It means that $90 \mathrm{ml}$ are absorbed by $3 \mathrm{~g}$ of the polymer (hydrogel optimal swelling degree for leachate is $1: 30$ ), while $310 \mathrm{ml}$ by 18 grams of fluff pulp (absorption rate of the fluff against leachate is 1:20).

The calculation of the total absorption capacity of the pad was made by applying the following formula:

$$
A_{t}=\left(P_{p}-P_{i}\right) \times A_{s}
$$

where:

- $A_{t}$ is the total absorption capacity;

- $P_{p}$ is the average weight of the individual absorbent pad;

- $P_{i}$ is the average weight of non-absorbent materials;

- $A_{s}$ is the specific capacity of absorption of the absorbent material.

The test has given good results, demonstrating applicability of superabsorbent polymers positioned within a pad. Innovation consists in transforming physical state of the waste itself, from a liquid status to a solid/stabilized one, without changing the chemical properties.

\section{References}

[1] Perillo G. \& Sorrentino F., Application of hydrogels in highly polluted affluents. WIT Conference IX Sustainable City, Siena (Italy), 2014.

[2] Canziani R. \& Cossu R., Modelli idrologici per la valutazione delle quantita di percolato e suo controllo. Atti del 30 Corso di aggiornamento in Ingegneria Sanitaria, 1985.

[3] Harsh D. C. \& Gehrke S. H., Characterization of ionic water absorbent polymers:determination of ionic content and effective crosslink density. In Absorbent Polymer Technology, L. Brannon-Peppas \& R.S. Harland Eds., Elsevier, Amsterdam, 1990.

[4] Aloys Huttermann, Lawrence J. B. Orikiriza \& Hillary Agaba, Application of Superadsorbent Polymers for improving the ecological chemistry of degraded or polluted lands. Clean Journal, Weinheim, 2009. 
[5] M. J. Zohuriaan-Mehr, Superadsorbent Polymer Materials. Iranian Polymer Journal, Iran, 2009.

[6] Gervasoni Sonia, Discariche controllate: significato e utilizzo delle discariche dopo l'uscita del decreto Ronchi (D.LGS. 22/97) e della nuova direttiva comunitaria (1999/31/CE). Hoepli, 2000.

[7] Sirini P., Ingegneria sanitaria-ambientale. Principi, teorie e metodi di rappresentazione, Mc-Graw Hill, 2002.

[8] M. Consiglio, V. Frenna \& S. Orecchio, Il laboratorio di chimica. Edises, 2001.

[9] Gregorio Crini, Recent developments in polysaccharide-based materials used ad adsorbents in wastewater treatment. Elsevier, France 2004.

[10] Yian Zheng, Yuntao Xie \& Aiqin Wang, Rapid and wide pH-independent ammonium-nitrogen removal using a composite hydrogel with threedimensional networks. Elsevier, China 2011.

[11] Jane Aiken \& Loo-Teck Ng, Calcium delivery using UV polymerized HEMA:NVP hydrogel in soil. 19th World Congress of Soil Science, Australia 2010. 